\title{
Highly efficient, tunable single photon source based on single molecules
}

\author{
Mathias Steiner, Achim Hartschuh, Rafał Korlacki, and Alfred J. Meixner ${ }^{\text {a) }}$ \\ Institut für Physikalische und Theoretische Chemie, Universität Tübingen, 72076 Tübingen, Germany
}

(Received 22 March 2007; accepted 11 April 2007; published online 4 May 2007)

\begin{abstract}
The authors studied spatially isolated terrylene molecules immobilized in a quasiplanar optical $\lambda / 2$-microresonator using confocal microscopy and spectroscopy at variable temperatures. At $T$ $=1.8 \mathrm{~K}$, they observed individual molecules relaxing into microresonator-allowed vibronic levels of their electronic ground state by emission of single fluorescence photons. Coupling the purely electronic transition of embedded molecules to the longitudinal photonic mode of the microresonator resulted in an ultimate spectral narrowing and an increased collection efficiency of the emitted single photon wave trains. (C) 2007 American Institute of Physics.
\end{abstract}

[DOI: 10.1063/1.2736294]

Single photon sources (SPSs) emitting optical wave trains that contain only one photon have recently attracted considerable scientific interest and they have numerous applications in spectroscopy and quantum optics. ${ }^{1}$ Individual dye molecules fulfill the necessary condition for true SPS: The intensity correlation measured from single molecule fluorescence exhibits photon antibunching, i.e., there is a diminishing probability for emission of two photons at the same time. ${ }^{2}$ Just recently, it has been reported that the zerophonon line (ZPL) emission of single dye molecules embedded in a crystalline matrix at cryogenic temperatures is a proper source of indistinguishable photons having ultralong coherence times in the range of nanoseconds.

The performance of a SPS based on a single molecule can still be improved essentially by utilizing a planar optical microresonator $^{5,6}$ and this technique has been used to enhance the radiative efficiency of single quantum dots, see, e.g., Refs. 7-9 and references therein. The modified photonic mode density in the presence of the microresonator is determined by the mirror spacing and can enhance ${ }^{10}$ or inhibit ${ }^{11}$ the spontaneous emission rate of embedded emitters. Under cryogenic conditions, the fluorescence spectra of dye molecules reveal their vibronic fine structure. Having the purely electronic transition of a single molecule on resonance with the longitudinal photonic (forward) mode of the microresonator, we expect to observe a suppression of redshifted transitions to excited vibronic levels of the molecular electronic ground state. This would result in a stream of highly directed single photons with an extremely narrow spectral distribution determined by the width of the ZPL, as it has been suggested already several years ago.,12

In this letter, we report optical studies of single terrylene molecules embedded in a quasiplanar optical $\lambda / 2$ microresonator that is easy to build, inexpensive, long term stable, and suitable for temperatures ranging from $T=300 \mathrm{~K}$ down to $T=1.8 \mathrm{~K}$.

In Fig. 1(a), a schematic of the microresonator is shown. Two silver mirrors $\mathrm{M}_{1,2}$ were separated by a layer of an UV-polymerizing optical adhesive (NOA 61, Norland) with slightly varying thickness $L(x, y)$ realized by applying a punctual force to one of the mirrors during the polymerization. ${ }^{13}$ The adhesive was doped with homoge-

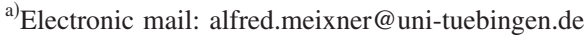

neously distributed and randomly oriented terrylene molecules $\left(c_{\mathrm{ter}} \approx 10^{-8} \mathrm{~mol} / \mathrm{l}\right)$. Light passing the microresonator in the $\lambda / 2$ regime obeys the transmission condition ${ }^{14,15}$

$$
L(x, y)=\left(1-\frac{\sum_{i} \Delta \phi_{i}\left(d_{i}, \vartheta, \lambda\right)}{2 \pi}\right) \frac{\lambda(x, y)}{2 n_{\mathrm{pol}} \cos \vartheta} .
$$

Here, $\Delta \phi_{i}$ denotes the phase change due to reflection at the respective silver mirror $i=1,2$ with thickness $d_{i}\left(d_{1}\right.$ $=30 \mathrm{~nm}, d_{2}=60 \mathrm{~nm}$; reflectivities $R_{1}=0.7, R_{2}=0.9$, and $\sum_{i} \Delta \phi_{i}=1.9$ at $\lambda=532 \mathrm{~nm}$ assuming normal incidence). The refractive index of the intracavity medium $n_{\text {pol }}$ is 1.56 . The incidence angle of a parallel light beam with respect to the $z$ axis is given by $\vartheta$. The transmission condition [Eq. (1)] yields the local mirror spacing $L(x, y)$ from the measured transmitted wavelength $\lambda(x, y)$. The mirror spacing variation $\Delta L(x, y) / \Delta x, y \simeq 10^{-3}$ ensures a planar resonator geometry within the focal diameter of our microscope objective (numerical aperture $=0.85$ ). The microresonator yields a cavity $Q$ around 50 in the $\lambda / 2$ regime and provides single molecule
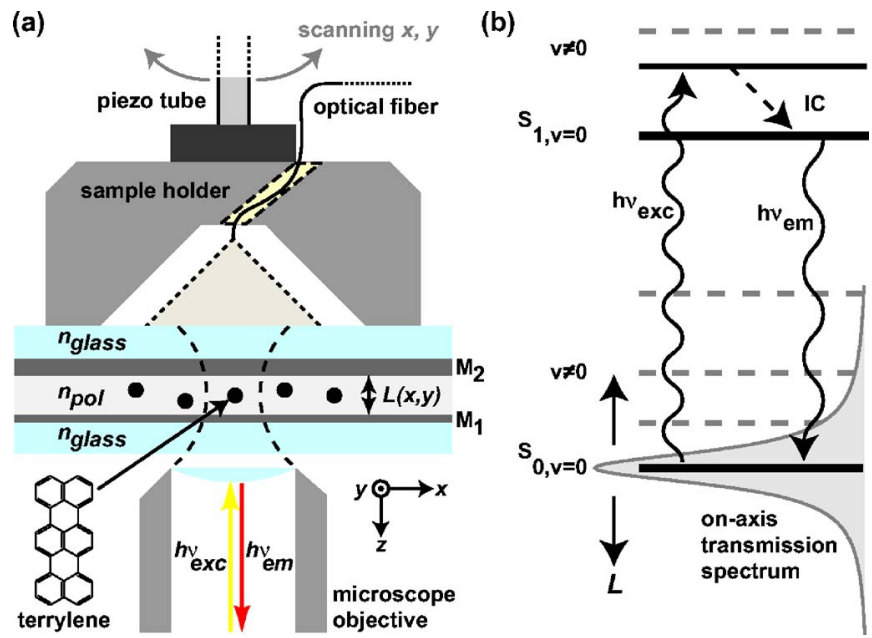

FIG. 1. (Color online) (a) Schematic diagram of the microscope head that is located in a bath cryostat. The microresonator consists of two silver mirrors $\mathbf{M}_{1,2}$ evaporated on glass coverslips. The polymer layer between $\mathbf{M}_{1,2}$ is doped with terrylene molecules. (b) Energy level diagram and excitationemission cycle of a fluorescent molecule embedded in the microresonator. Intersystem crossing to a dark molecular triplet state is neglected. 


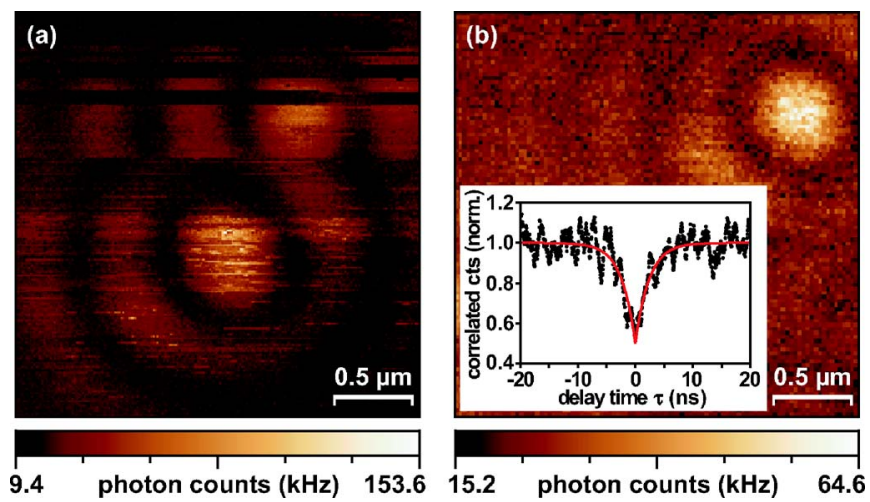

FIG. 2. (Color online) Diffraction-limited confocal fluorescence microscopy images of individual terrylene molecules embedded in a $\lambda / 2$ microresonator at $T=1.8 \mathrm{~K}$. (a) Two terrylene molecules spatially separated by around $1 \mu \mathrm{m}$ exhibit strong intensity fluctuations. (b) Single molecule delivering stable fluorescence emission. (Inset) The corresponding intensity correlation measurement (circles) exhibits photon antibunching. Fitting the model function $1-a \exp \left\{-|\tau| / \tau_{\text {meas }}\right\}$ to the data (line) reveals an excited state lifetime $\tau_{\text {meas }}=(2.3 \pm 0.2) \mathrm{ns}$.

sensitivity over months without noticeable degradation of optical quality. $^{13}$

For optical experiments at variable temperatures, the microresonator was mounted on a piezoelectric tube scanner, and an optical fiber was guided into the sample holder to allow for transmission measurements [see Fig. 1(a)]. For confocal imaging, we used a single mode ring dye laser (CR699 with R6G-solution, Coherent) operated around $570 \mathrm{~nm}$ and an avalanche photon counting module (SPCM-AQ 141, EG \& G) as detector. Fluorescence spectra were acquired with a grating spectrograph (SpectraPro 300i, Acton Research) equipped with a charge coupled device camera (LNCCD-1340/100-EB/1, Princeton Instruments). Intensity correlations ${ }^{1}$ were measured using a Hanbury-Brown and Twiss setup consisting of a beam splitter cube, two avalanche photon counting modules (SPCM-AQR-14, Perkin-Elmer), and a time correlated single photon counting board (timeharp 100, Picoquant).

The excitation-emission cycle for a dye molecule embedded in the microresonator is depicted in Fig. 1(b): We use the vibronic excitation scheme ${ }^{3}$ to prepare single molecules into excited vibronic levels of the first excited electronic state, i.e., $S_{0, v=0} \rightarrow S_{1, v \neq 0}$. The $S_{1, v=0}$ level is reached by fast nonradiative internal conversion (IC) of vibrational energy on a time scale of picoseconds accompanied by a loss of coherence between the excitation field and the molecular transition dipole. The efficiency of a radiative transition to a vibronic level of the molecular electronic ground state $S_{0}$ depends on the mirror spacing $L(x, y)$ that can be determined from the measured local on-axis transmission spectrum indicating the forward mode of the microresonator. In Fig. 1(b), the on-axis transmission spectrum is on resonance with the purely electronic transition $S_{1, v=0} \rightarrow S_{0, v=0}$ that corresponds to the strongest and narrowest fluorescence line in the measured spectra, i.e., the ZPL.

In Fig. 2, confocal microscopy images show the integrated fluorescence emission of individual terrylene molecules embedded in the $\lambda / 2$ microresonator at $T=1.8 \mathrm{~K}$. We observed single molecules showing fluorescence intensity fluctuations in the range of milliseconds to seconds [see Fig. $2(\mathrm{a})$ ] as well as irreversible photobleaching. We also found stable emitters [see Fig. 2(b)] allowing hours of observation.

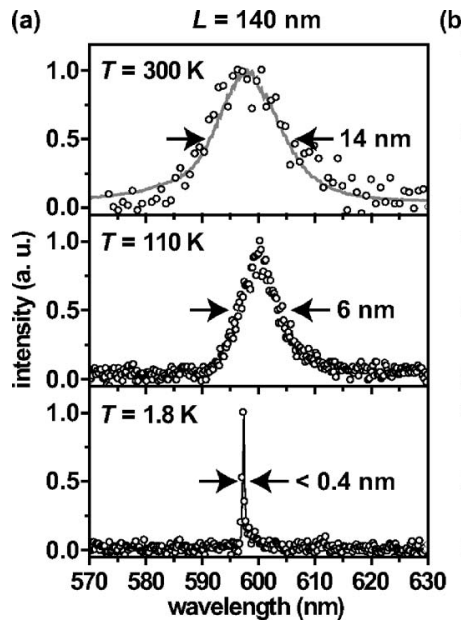

(b) $\quad T=1.8 \mathrm{~K}$

FIG. 3. (Color online) (a) Influence of the temperature $T$ on microresonatorcontrolled fluorescence spectra of single terrylene molecules (rings) for a constant mirror spacing $L=140 \mathrm{~nm}$. The spectral full width at half maximum values are indicated by arrows. A measured on-axis transmission spectrum of the microresonator is shown as a gray line. (b) Influence of the mirror spacing $L$ on microresonator-controlled fluorescence spectra of single terrylene molecules (rings) for constant $T=1.8 \mathrm{~K}$. The coupling efficiency for specific electronic-vibronic transitions is determined by the mirror spacing $L_{1}>L_{2}>L_{3}$. The angular distribution of the emitted light for the transitions labeled I, II, and III is indicated in the corresponding microresonator schematics and given by Eq. (1).

The intensity correlation detected for the isolated terrylene molecule imaged in Fig. 2(b) is shown in the inset. The dip at $\tau=0 \mathrm{~ns}$ is a clear evidence for photon antibunching demonstrating observation of a single emitter and emission of single fluorescence photons. ${ }^{1,2}$

The influence of the temperature on microresonatorcontrolled fluorescence spectra of single terrylene molecules is shown in Fig. 3(a). At room temperature $(T=300 \mathrm{~K}$, upper panel), the spectral shape of the fluorescence matches the local on-axis transmission spectrum of the microresonator, and the spectral width of the emission is reduced by up to one order of magnitude as compared to single molecule fluorescence in free (nonconfined) space. ${ }^{13}$ At $T=110 \mathrm{~K}$ (middle panel), the fluorescence emission is already significantly narrowed below the spectral width of the cavity resonance. Finally, at $T=1.8 \mathrm{~K}$ (lower panel), the fluorescence emission is dominated by a spectrally isolated ZPL as narrow as the instrument response function of our spectrometer.

The influence of the mirror spacing on the fluorescence spectra of single terrylene molecules is shown in Fig. 3(b) for three different molecules. Three representative transitions are labeled by roman numerals: The ZPL (I) was set to be the origin $\tilde{\nu}=0 \mathrm{~cm}^{-1}$ of the relative wave number scale. The transition to the molecular electronic ground state accompanied by excitation of a molecular vibration $S_{1, v=0} \rightarrow S_{0, v \neq 0}$ is marked by II for the strong long-axis stretch vibration of the molecule around $\tilde{\nu}=250 \mathrm{~cm}^{-1}$ and III for the weak ring breathing vibration at $\widetilde{\nu}=850 \mathrm{~cm}^{-1}$. ${ }^{16}$ For molecule 1 (upper panel), the weak vibronic transition labeled III is efficiently detected since it is coupled to the forward mode of the microresonator, i.e., the on-axis resonance $(\vartheta=0)$, as indicated in the corresponding microresonator schematic. Additionally, the molecule couples to blueshifted off-axis resonances $(\vartheta>0)$ with a reduced detection efficiency. By reducing $L$, we observed that vibronic lines in the measured spectra of detected molecules were cut successively from the 
red side of the spectrum: For molecule 2 at $L_{2}$ (middle panel), only the vibronic line corresponding to the long-axis stretch vibration around $\widetilde{\nu}=250 \mathrm{~cm}^{-1}$ (II) is visible in the spectrum. The purely electronic transition (I) is still coupled to an off-axis resonance of the microresonator. Further reducing the mirror spacing to $L_{3}$ allows addressing molecule 3 (lower panel). Here, the strong ZPL (I) is on resonance with the forward mode of the microresonator and remains as the only significant feature in the measured spectrum.

In order to reveal the coherence properties of single photon streams leading to spectra as shown in the lower panels of Figs. 3(a) and 3(b), we performed two-beam interferometric experiments. ${ }^{17}$ We found that the temporal coherence is strongly perturbed by photons associated with the spectrally broad phonon wing directly following the ZPL on the red side of the spectrum. The phonon wing appears with a small amplitude in the lower spectrum in Fig. 3(b) and cannot be suppressed efficiently by our microresonator. To spectrally isolate the ZPL and the phonon wing, the cavity $Q$ would have to be increased by several orders of magnitude, e.g., utilizing high- $Q$ dielectric microcavities. ${ }^{7}$

We presented a microresonator-enhanced SPS based on single terrylene molecules operated at variable temperatures. The photons emitted into the forward mode of the microresonator do not walk off after multiple internal reflections and can be efficiently collected with a microscope objective or coupled to optical fibers. Coupling the ZPL emission of a single molecule to a single cavity mode can be considered as an important step towards realization of a single molecule dye laser. ${ }^{18}$
The authors acknowledge technical support by G. Schulte (Universität Siegen, Germany) and financial support by the Research Center for Micro- and Nanochemistry and Engineering (Universität Siegen, Germany) and the Deutsche Forschungsgemeinschaft (Me 1600/6-1/2).

${ }^{1}$ B. Lounis and M. Orrit, Rep. Prog. Phys. 68, 1129 (2005).

${ }^{2}$ W. E. Moerner, New J. Phys. 6, 88 (2004).

${ }^{3}$ A. Kiraz, M. Ehrl, C. Bräuchle, and A. Zumbusch, Appl. Phys. Lett. 85, 920 (2004).

${ }^{4}$ A. Kiraz, M. Ehrl, T. Hellerer, Ö. E. Müstecaplioğlu, C. Bräuchle, and A. Zumbusch, Phys. Rev. Lett. 94, 223602 (2005).

${ }^{5} \mathrm{H}$. Yokoyama and K. Ujihara, Spontaneous Emission and Laser Oscillation in Microcavities (CRC, Boca Raton, FL, 1995).

${ }^{6}$ R. K. Chang and A. J. Campillo, Optical Processes in Microcavities (World Scientific, Singapore, 1996).

${ }^{7}$ K. J. Vahala, Nature (London) 424, 839 (2003).

${ }^{8}$ A. J. Bennett, D. C. Unitt, P. See, A. J. Shields, P. Atkinson, K. Cooper, and D. A. Ritchie, Appl. Phys. Lett. 86, 181102 (2005).

${ }^{9}$ G. Ramon, U. Mizrahi, N. Akopian, S. Braitbart, D. Gershoni, T. L. Reinecke, B. D. Gerardot, and P. M. Petroff, Phys. Rev. B 73, 205330 (2006).

${ }^{10}$ E. M. Purcell, Phys. Rev. 69, 681 (1946).

${ }^{11}$ D. Kleppner, Phys. Rev. Lett. 47, 233 (1981).

${ }^{12}$ C. Brunel, B. Lounis, P. Tamarat, and M. Orrit, Phys. Rev. Lett. 83, 2722 (1999).

${ }^{13}$ M. Steiner, F. Schleifenbaum, C. Stupperich, A. V. Failla, A. Hartschuh, and A. J. Meixner, ChemPhysChem 6, 2190 (2005).

${ }^{14}$ M. Born and E. Wolf, Principles of Optics, 6th ed. (Pergamon, Oxford, 1980).

${ }^{15}$ H. Becker, S. E. Burns, N. Tessler, and R. H. Friend, J. Appl. Phys. 81, 2825 (1997)

${ }^{16}$ A. B. Myers, P. Tchénio, M. Z. Zgierski, and W. E. Moerner, J. Phys. Chem. 98, 10377 (1994).

${ }^{17}$ R. Korlacki, M. Steiner, H. Qian, A. Hartschuh, and A. J. Meixner, Chem. Phys. Chem., published online 10 April 2007 (DOI 10.1002/cphc200716).

${ }^{18}$ Z. S. Wang, H. A. Rabitz, and M. O. Scully, Laser Phys. 15, 118 (2005). 